\title{
Aligned Electrospun Cellulose Scaffolds Coated with rhBMP-2 for Both in vitro and in vivo Bone Tissue Engineering
}

The Authors: Ximu Zhang, Chao Wang, Min Liao, Lina Dai, Yingying Tang, Hongmei Zhang, Phil Coates, Farshid Sefat, Liwen Zheng, Jinlin Song, Zhuo Zheng, Dan Zhao, Maobin Yang*\$, Wei Zhang*+, and Ping Ji

\section{Authors' information:}

Ximu Zhang ${ }^{1,2}$, ChaoWang ${ }^{1}$, Min Liao ${ }^{3}$, Lina Dai ${ }^{1}$, Yingying Tang ${ }^{1}$, Hongmei Zhang ${ }^{1}$, Phil Coates $^{4,5}$, Farshid Sefat ${ }^{4,5}$, Liwen Zheng ${ }^{1}$, Jinlin Song ${ }^{1}$, Zhuo Zheng ${ }^{7}$, Dan Zhao ${ }^{1}$, Maobin Yang $*+^{6}$, Wei Zhang $*+^{7}$ and Ping $\mathrm{Ji}^{1}$

1, Chongqing Key Laboratory of Oral Disease and Biomedical Sciences \& Chongqing Municipal Key Laboratory of Oral Biomedical Engineering of Higher Education \& Stomatological Hospital of Chongqing Medical University, Chongqing, 401174, China.

2, State Key Laboratory of Oral Diseases \& National Clinical Research Center for Oral Diseases \& Dept. of Preventive Dentistry, West China Hospital of Stomatology, Sichuan University, Chengdu, 610041, China.

3, Children's Hospital of Chongqing Medical University, Chongqing, 400014, China

4, Biomedical and Electrical Engineering Department, School of Engineering, University of Bradford, Bradford, UK.

5, Interdisciplinary Research Centre in Polymer Science \& Technology (IRC Polymer), University of Bradford, Bradford, UK.

6, Regenerative Health Research Laboratory, Department of Endodontology, Kornberg School of Dentistry, Temple University, Philadelphia 19140, USA.

7, State Key Laboratory of Polymer Materials Engineering, Polymer Research Institute at Sichuan University, Chengdu, 610065, China.

\section{AUTHOR INFORMATION}

*: The corresponding authors

Wei Zhang E-mail: weizhang@scu.edu.cn

Maobin Yang E-mail: myang@dental.temple.edu

Author Contributions

+ These authors contributed equally.

Notes: The authors declare no competing financial interest. 
ABSTRACT

Physical properties of scaffolds such as nanofibers and aligned structures have been reported to exert profound effects on the growth and differentiation of stem cells due to their homing-effect features and contact guidance. However, the biological function of aligned nanofiber utilized as bone-scaffold has not been rigorously characterized. In the present study, aligned electrospun cellulose/CNCs nanocomposite nanofibers (ECCNNs) loaded with bone morphogenic protein-2 (BMP-2) were used for the first time to investigate (1) in vitro osteogenic differentiation of human mesenchymal stem cells (BMSCs) and (2) in vivo collagen assembly direction and cortical bone regeneration. Aligned ECCNNs scaffolds loaded with BMP-2 possess good biological compatibility. The growth orientation of BMSCs followed the underlying aligned nanofibers morphology, accompanied with increased alizarin red stain, ALP activity and calcium content in vitro while, a rabbit calvaria bone defect model was used in an in vivo study.

KEYWORDS: Electrospinning; cellulose; cellulose nanocrystals; aligned nanofibers; collagen assembly; cortical bone;

\section{INTRODUCTION}

Cellulose, categorized as a linear polysaccharide, represents about 1.5 trillion tons of the total annual biomass production which has been considered as one of the world's most abundant natural and renewable resource of raw material for the increasing demand of environmentalfriendly and biocompatible products (Ma, Qing, Li, Zhu, Fu \& Sun, 2013). Harvesting nanostructure from natural, sustainable, and earth-abundant raw materials is one of the goals in the next decades. Recently, the production of nanocellulose biomaterials has received increasing attention due to their high strength and stiffness combined with small size, low density, renewability and biodegradability (O'Donnell, Okkelman, Timashev, Gromovykh, Papkovsky \& Dmitriev, 2018). Particularly, the low water solubility and hydrophilicity of cellulose allow for good control over scaffold fabrication and design (Nada, Abdellatif, Ali, Abdelazeem, Soliman \& Abou-Zeid, 2018). Cellulose-based biomaterials applying in vivo have demonstrated only negligible inflammatory response reactions and good biocompatibility.

During the past decade, electrospinning (Zong et al., 2018, Moztarzadeh, 2018, Sefat, 2018a, Tariverdian, 2018, Sefat, 2018b, Urbanska, 2018, Raja, 2018) has emerged as a promising tool in tissue engineering because it is easy to produce fibrous structures with diameters ranging from dozens of nanometers to a few hundred nanometers (Zarrintaj, 2018, Sefat, 2016, Mahjour, 2016), and some physical properties such as fiber alignment and fiber diameter can be precisely controlled by changing the spinning parameters (Kishan \& Cosgriff-Hernandez, 2017, Sefat, 2015). The thin, continuous polymer fibers generated by electrospinning have micro to nanoscale $(<100 \mathrm{~nm})$ topography and high porosity $(>90 \%)$, which is similar to the natural extracellular matrix (ECM), promoting cellular interactions, guiding cell growth and resulting in new tissue formation (Yang, Li, He, Ma, Ni \& Zhou, 2018). However, electrospun nonwoven nanofibers usually exhibit poor mechanical strength, representing one of the limitations for the materials (Sehaqui, Morimune, Nishino \& Berglund, 2012). In 2006, Thomas et al (Thomas, Jose, Chowdhury, Sullivan, Dean \& Vohra, 2006) reported the scaffold of electrospun polycaprolactone ( $P C L$ ) nanofibers could achieve a high degree of alignment and orientation when gathered on a fast rotating drum, leading to several-fold increase of the mechanical 
strength along the fiber orientation direction (Raja, 2018).

Numerous polymer nanofibers have been fabricated using electrospinning, but electrospun nanofibers from native cellulose has rarely been reported mainly because cellulose cannot be directly dissolved in common solvents owing to its high crystallinity enhanced by tremendous hydrogen bonding network (Wake, Patrick \& Mikos, 1994; Wang, Zhang, Zhang, Li, Yu \& Lin, 2013). Alternatively, most researches on cellulose electrospinning are based on cellulose derivatives (Du \& Hsieh, 2009; Frey, 2008) and the resultant electrospun fibers are usually very weak in tensile strength ( $<1 \mathrm{MPa}$ (Suwantong, Opanasopit, Ruktanonchai \& Supaphol, 2007). There are limited kinds of solvents that can dissolve cellulose (Jayaramudu, Reddy, Varaprasad, Sadiku, Sinha Ray \& Varada Rajulu, 2013) and so far, only a few of them, namely, N-methylmorpholine $\mathrm{N}$-oxide solution/water $\left(\mathrm{NMMO} / \mathrm{H}_{2} \mathrm{O}\right)$, lithium chloride/dimethyl acetamide (LiCl/DMAc) and ionic liquids, have been successfully applied for electrospinning of native cellulose mainly due to the dielectric requirements of this technique (Magalhaes, Cao \& Lucia, 2009).

To address the weak strength of electrospun nanofibers, some stiff nanoparticles have been blended into the solutions for electrospinning. Cellulose nanocrystals (CNCs) with cellulose I crystal structure have attracted considerable attention in the nanocomposite field (He et al., 2014). The theoretical Young's modulus for ideal CNCs is estimated to be $167.5 \mathrm{GPa}$ and elastic moduli of CNCs from tunicate and cotton have been experimentally confirmed to be up to 105 and $143 \mathrm{GPa}$, respectively (Podsiadlo, Choi, Shim, Lee, Cuddihy \& Kotov, 2005). These excellent mechanical properties have made CNCs very promising as an effective reinforcement for polymer nanocomposites (He et al., 2014). Additionally, a hybrid of nanoparticles and biomaterials sometimes provides extra effects for tissue engineering applications (Bertrand, Grenier \& Mahmoudi, 2017; Zhao, Lu, He, Zhang, Zhang \& Zhang, 2015).

Many researchers have explored nanofiber scaffolds in tissue engineering for the regeneration of skin (J. Bye, Bullock, Singh, Sefat, Roman \& Macneil, 2014; Mahjour, Fu, Yang, Fong, Sefat \& Wang, 2015), nerve (Mohamadi et al., 2017; Mohammadi et al., 2018), cornea (Deshpande et al., 2013, Ortega et al., 2014), cartilage (Daghigh Ahmadi et al., 2018; Raja et al., 2018; Sefat, 2017), oral (Nejatian et al., 2017), adipose tissue (Amini et al., 2018) and bone (Sefat, Denyer \& Youseffi, 2011 , 2014). Among them, aligned nanofibers have become a popular platform for in vitro tissue engineering attempts due to that they structurally mimic native tissue organization (Driscoll, Sun, Guven, Fourkas \& Losert, 2014). Up to date, most studies reported that repairing bone defect by tissue engineering increased the volume of sponge bone rather than cortical bone, though the latter is more significant in loading-bear site or dental implant than the former. Our previous study (He et al., 2014) has electrospun uniaxially aligned cellulose nanofibers with well oriented CNCs embedded. This kind of electrospun cellulose/CNCs nanocomposite nanofibers (ECCNNs) has four main advantages in tissue engineering: 1) The raw material is cellulose coming from cotton, which is cost effective, 2) provide aligned structure of nanofibers, 3) and biocompatible. The degradable product of cellulose is glucose, which has no toxicity; and 4) cellulose nanocrystals (CNCs) remarkably enhance the mechanical strength of the scaffolds. However, the effect of well-defined geometries from nanofibers of scaffold on in vivo tissue formation, particularly in bone healing process, has not yet been fully investigated (Murphy, O'Brien, Little \& Schindeler, 2013). In this study, we loaded rhBMP-2, which is capable to promote osteogenic differentiation, onto the fabricated aligned ECCNNs. The objectives of this study are to test whether the aligned ECCNNs loaded with BMP-2 could induce aligned calcium nodules in vitro, as well as to test the mimicking ECM structure to recruit stem cells in vivo and generate aligned collagen and cortical bone. 


\section{Materials and Methods}

\subsection{Materials}

Purified cotton (Xuzhou Health Materials Co., Ltd) was used as the raw material. Analytical grade $\mathrm{N}, \mathrm{N}$-dimethylacetamide (DMAC) and lithium chloride (LiCl) were purchased from Chengdu Kelong Chemical Plant. For cell culture study, Dulbecco's modified eagle's medium (DMEM), trypsin, fetal bovine serum (FBS), Dulbecco's Modified Eagle's medium (DMEM), Triton-X 100, F12, 40,6-diamidino-2-phenylindole (DAPI), rhBMP-2 ELISA kit, Masson Trichrome kit, Alkaline Phosphatase (ALP) Activity kit and antibiotics (100 U/mL penicillin-streptomycin) were purchased from Sigma Chemical Co. (St. Louis, MO, USA); Alexa-fluor 568 antibodies were purchased from Invitrogen (Molecular probes, Carlsbad, CA, USA). Fetal bovine and trypsin were purchased from Gibco BRL (Life Technologies, Grand Island, NY). rhBMP-2 was purchased form Humanzyme (Chicago, IL). Glutaraldehyde solution (25\%, in weight) was obtained from Zhong-shan FineChem Ltd. (Guangdong, China). MTS assay kit (Promega ${ }^{\text {TM }}$ CellTiter $96^{\text {TM }}$ AQueous Nonradioactive Cell Proliferation Assay Kit, Promega TM G1111) was purchased from Promega Ltd. (Shanghai, China). Alizarin Red S staining kit and Calcium assay kit were purchased from Kanto Chemical (Tokyo, Japan). All the other reagents used were of analytical grade and were used without further purification. Distilled water was used throughout the experiment.

\subsection{Scaffold Fabrication and rhBMP-2 loading}

Aligned and unaligned/random scaffolds were fabricated using the electrospinning technique as previously reported (He et al., 2014). Briefly, CNCs were prepared following a hydrolysis method (Podsiadlo, Choi, Shim, Lee, Cuddihy \& Kotov, 2005; Podsiadlo et al., 2007). Then the CNCs were dispersed in DMAc through a solvent exchange process. Cotton was "activated" and then dissolved in the LiCl/DMAC ( $8: 92$ by weight) solution under magnetic stirring to obtain the $2 \mathrm{wt} \%$ cellulose solution. The cellulose/CNCs spinning dope was prepared by mixing the CNCs suspension with the cellulose solution. A computer controlled automatic electrospinning equipment (FM-12, Fuyouma Technology Co., Beijing, China) was used to fabricate ECCNNs at 20 $\mathrm{kV}$ voltage, $0.03 \mathrm{~mL} / \mathrm{min}$ flow rate of the solution. A steel rotating collector $(6 \mathrm{~cm}$ in diameter) wrapped with aluminum foil was placed $10 \mathrm{~cm}$ away from the tip of the nozzle. The tangential velocity of the rotating collector was set at $300 \mathrm{~m} / \mathrm{min}$ and $50 \mathrm{~m} / \mathrm{min}$ to produce the aligned and random nanofibers, respectively. The rotating collector was partly immersed in a water coagulation bath to thoroughly remove the solvent from electrospun fibers and produce dimensionally stable fibers. The obtained nonwovens were subsequently dried under ventilation and then kept in a desiccator. A $20 \%$ loading (in weight) of CNCs was used in this study since it could increase the tensile strength and elastic modulus of ECCNNs along the fiber alignment direction by 101.7 and $171.6 \%$, respectively, as we studied before (He et al., 2014).

To load rhBMP-2 on the ECCNN scaffold, a circular scaffold (diameter $=8 \mathrm{~mm}$ ) was fixed on a spin-coating machine with a bovine collagen solution as the delivery vehicle. The rhBMP-2 doses were chosen according to the literature (Filion, Skelly, Huang, Greiner, Ayers \& Song, 2017). Briefly, $40 \mu \mathrm{l}$ bovine collagen solution $(3 \mathrm{mg} / \mathrm{mL}$ ) was mixed with $20 \mu \mathrm{lhBMP}-2$ solution $(5 \mathrm{mg} / \mathrm{mL})$ in a sterile centrifuge tube. For each loading, $20 \mu \mathrm{l}$ of mixed liquid was added to the center of the scaffolds $(4000 \mathrm{rpm}, 20 \mathrm{~s})$. To load enough rhBMP-2, this process was repeated for three times (Perikamana et al., 2015). After that, the central $5 \times 5 \mathrm{~mm}^{2}$ 
square was cut by a sterilized scissors. All the aforementioned procedures were performed in an aseptic manner and the rhBMP-2 loaded scaffolds were freshly prepared and stored at $4{ }^{\circ} \mathrm{C}$ before use.

The morphologies of the electrospun fibers were observed on a field-emission scanning electron microscope (SEM, Inspect F50, FEI) at $20 \mathrm{kV}$. ImageJ software was used for the analysis of diameter distributions of more than 100 electrospun fibers selected randomly from the SEM images. The specific surface area and the pore volume were calculated by Brunauer-EmmettTeller (BET) and Barrett-Joyner-Halenda (BJH) methods, respectively. The BMP-2 release was measured by ELISA kit (Simson, Strehin, Lu, Uy \& Elisseeff, 2013).

\subsection{Isolation and culture of bone marrow-derived Stem cells (BMSCs)}

This study was designed in conformity to the ARRIVE (Animal Research: Reporting of In Vivo Experiments) guidelines and approved by the Ethics Committee of West China School of Stomatology, Sichuan University (approval number WCCSIRB-D-2015-073), specific pathogenfree Wistar rats weighing 80-100 g (male) were purchased from the Laboratory Animal Center of Sichuan University (Chengdu, Sichuan Province, China). The animals were sacrificed by anesthesia with $5 \%$ isoflurane. The femurs and tibias were harvested and the metaphysis on both sides was removed using a rongeur under sterile conditions. Bone marrow was collected by flushing the femur and tibia with medium. Following centrifugation at $150 \mathrm{~g}$ for $8 \mathrm{~min}$ at $25^{\circ} \mathrm{C}$, the cell pellets were mixed thoroughly with DMEM/F12 supplemented with $10 \%$ fetal bovine serum and antibiotics cultured in $5 \% \mathrm{CO}_{2}$ at $37^{\circ} \mathrm{C}$. Medium changes were made every $3-4$ days. Sub-confluent cell monolayers were dissociated using $0.25 \%$ trypsin and cells were sub-cultured to passage 2-4 for the following experiments.

\subsection{Cell Seeding, growth and osteo-Differentiation}

Generally, scaffolds were cut by a self-made metal punch with $15 \mathrm{~mm}$ diameter, which matched the 24 well cell culture plate ( $15.6 \mathrm{~mm}$ diameter). $100 \mu \mathrm{l}$ BMSCs suspension at a density of $1 \times 10^{6}$ cells $/ \mathrm{mL}$ was dropped at the center of the scaffold and cultured in cell incubator in $5 \% \mathrm{CO}_{2}$ at $37^{\circ} \mathrm{C}$ for 4 hours to allow cells adhere to scaffold not to well of culture plate. After that, $1 \mathrm{~mL}$ osteogenic medium (DMEM media supplemented with $300 \mu \mathrm{M}$ L-ascorbic acid sodium salt, 10 $\mathrm{nM}$ dexamethasone, $10 \mathrm{mM}$-glycerophosphate, $10 \%$ fetal bovine serum, and $1 \%$ penicillin/streptomycin) was added. The culture medium was changed every 2 days.

Cells growth and viability and distribution among the scaffolds were assessed using the MTS assay at day 1, 3, 5, 7. After BMSCs were seeded on scaffold in 96 well plate, DMEM containing antibiotic solution and $10 \%$ fetal bovine serum was used. Cell culture medium was changed every 2 days. At day 1, 3, 5, 7, culture medium containing MTS solution was added followed by incubation for $4 \mathrm{~h}$ at $37^{\circ} \mathrm{C}$. And after $3 \mathrm{~h}$ incubation, the absorbance at $490 \mathrm{~nm}$ was measured using a spectrophotometer (Shimadzu, Kyoto, Japan). 
paraformaldehyde for 20 min, then permeabilized in PBS containing 10 vol\% FBS plus 0.5 vol\% Triton-X 100 for $20 \mathrm{~min}$, and incubated in PBS containing 10 vol\% FBS for $30 \mathrm{~min}$. Thereafter, samples were stained with Alexa-fluor 568 Conjugated Phalloidin for filamentous actin fluorescence (1:200) and cell nuclei were marked by DAPI staining for nuclei UV-visualization (1:2000) for 2 h. Subsequently, specimens were thoroughly washed with PBS, and rinsed with deionized water for 2 min. Finally, specimens were visualized using an Olympus FV1000 confocal laser scanning microscope (Olympus, Tokyo, Japan) at $460 \mathrm{~nm}$ (emission) and the multitrack images were captured with a 60x/1.35 NA objective.

\subsection{Osteogenic mineralization in vitro}

After BMSCs were co-cultured with scaffolds in osteogenic medium, Alkaline Phosphatase (ALP) Activity Measurement, Calcium assay and Alizarin Red $S$ staining were done to quantitatively evaluate the osteo-differentiation effect.

Briefly, after 14 and 21 days of osteogenic differentiation, cultured scaffolds were cut into small pieces by scissors, sonicated and centrifuged. Supernatant ALP activity at each time-point was determined with substrate solution (SIGMA FASTTM p-nitrophenyl phosphate and Tris buffer tablets, Sigma) by spectrophotometer. Absorbance reading was taken at $405 \mathrm{~nm}$. Meantime, after 14 and 21 days, those electrospun scaffolds were washed in PBS and were used to determine calcium content. Calcium was extracted with $0.5 \mathrm{~mL} 5 \%$ trichloroacetic acid for $30 \mathrm{~min}$ and calcium content of the supernatant was determined using creso phthalein complex one (Teco Diagnostics). The absorbance of samples was measured at $575 \mathrm{~nm}$, followed by the protocol of the kit.

Alizarin Red S staining of calcium deposits was done to assess the aligned mineralization of cell layers. It was done following the study described previously (Dadsetan et al., 2015). Briefly, the samples were collected after 28 days, fixed with $10 \%$ formalin solution for $30 \mathrm{~min}$. 1\% Alizarin Red $\mathrm{S}$ stain solution $(\mathrm{pH} 4.3)$ was added to each well and incubated for 20 min at room temperature. The alizarin dye was extracted using $300 \mu \mathrm{L} 50 \%$ acetic acid solution. The plates were placed onto a rocker for $10 \mathrm{~min}$ to allow for complete dissolution of the dye. The solution was then placed into $1.5 \mathrm{~mL}$ Eppendorf tubes and vortexed for $30 \mathrm{~s}$. The slurry was then centrifuged and supernatant was added to $10 \%$ ammonium hydroxide to bring the $\mathrm{pH}$ to 4.1 . Triplicates of $100 \mu \mathrm{L}$ were placed into a 96-well plate and the absorbance was read at $405 \mathrm{~nm}$. After that, scaffolds were rinsed in PBS for another three times and imaged with a microscope.

\subsection{Animal study for bone regeneration in vivo.}

After receiving approval from the Ethics Committee of West China School of Stomatology, Sichuan University (Approval number WCCSIRB-D-2015-073), adult female New Zealand white rabbits (West China Laboratory Animal Center) of 7 months old weighting 2-2.5 kg (Dashuo Laboratory Animals Inc, Chengdu China) were used in this study $(n=12)$. All animal surgeries in this study were conducted in compliance with the Animal Welfare Act, the implementing Animal Welfare Regulations, and the principles of the Guide for the Care and Use of Laboratory Animals. To assess the healing potential of the aligned or random scaffolds with rhBMP-2 $(50 \mu \mathrm{g} / \mathrm{mL})$, a $5 \times 5 \mathrm{~mm}$ square-sized defect was created in each side of the adult New Zealand white rabbit cranial bone. Scaffolds were cut into $5 \times 5 \mathrm{~mm}$ square size and placed into the bone defect area. After that, soft tissues were closed in three different layers with resorbable 3-0 sutures. The 
rabbits were recovered and survived for 12 weeks. Then the calvarial samples containing the healed defects were harvested post euthanasia and fixed in $10 \%$ formalin.

Then each specimen was placed on the scanning platform of a Micro-CT ( $\mu 80$, Skyscan, Kontich, Belgium). Micro-CT was performed using a model 1076 apparatus operating with an anode electrical current of $100 \mathrm{kV}, 500 \mathrm{~mA}$ at a resolution of $6 \mu \mathrm{m}$. The regions of interest (ROI) that included the scaffold and bone defect area, around bone were also selected. After scanning, three-dimensional (3D) models were generated by CTVol (Skyscan), and bone volume and density in bone defect area were analyzed by the CTAn program (Skyscan), which was also used to examine the Micro-CT data sets for new bone growth. The volume of interest (VOI) consisted of the collective sum of all ROI layers over continuous set of cross-sectional image slices. This represents the regenerated bone only. Further, new bone volume and bone mineral density in bone defect area was calculated by the phantom and Housefield units [HU]. A bone threshold value in CT HU was determined between low phantom (0.25) $1089.7966 \mathrm{HU}$ and high phantom (0.75) $3241.0643 \mathrm{HU}$ (Zhang et al., 2014). Based on this threshold, a distinction between bone and no bone volume elements can be made. As the volume of ROI corresponds to the volume of original bone, the amount of bone present within the ROI is considered as the new formed bone. To create 3D images binary threshold (Gray scale index, bone defect area: $50 \times 50 \mathrm{~mm}$ ) were selected.

After Micro CT scanning, samples were immersed in $10 \%$ phosphate-buffered formalin, decalcified with $10 \%$ EDTA at pH 7.4 for 3 months at $4^{\circ} \mathrm{C}$ with a weekly change of solution, then samples were dehydrated, cleared and subsequently embedded in paraffin. Tissue sections, 4 $\mu \mathrm{m}$ in thickness, were cut using a Leica RM 2265 microtome and mounted on glass slides and subjected to H\&E and Masson Trichrome staining. Images of histology were scanned by Virtual microscopy solutions (Aperio Technologies Co. the U.S) and viewed by Image Scope viewing software (Leica Biosystems Co. v11.2.0.780).

\subsection{Statistical analysis}

Data were collected from triple duplicated samples and all data were represented as mean \pm standard error of the mean. All results of the control and experimental groups were analyzed independently. We used one-way ANOVA to examine the differences in the variables among different experimental conditions. $p<0.05$ was considered as statistically significant.

For the quantification of data, each assay was repeated at least 3 times independently. The results of the quantitative analyses were expressed as the means \pm standard error of mean. The means were compared using one-way analysis of variance (one-way ANOVA) followed by Tukey's post-hoc assuming equal variances with the SPSS 17 (SPSS, Inc., Chicago, IL, USA). A value of $\mathrm{P}<0.05$ was considered to indicate a statistically significant difference. 


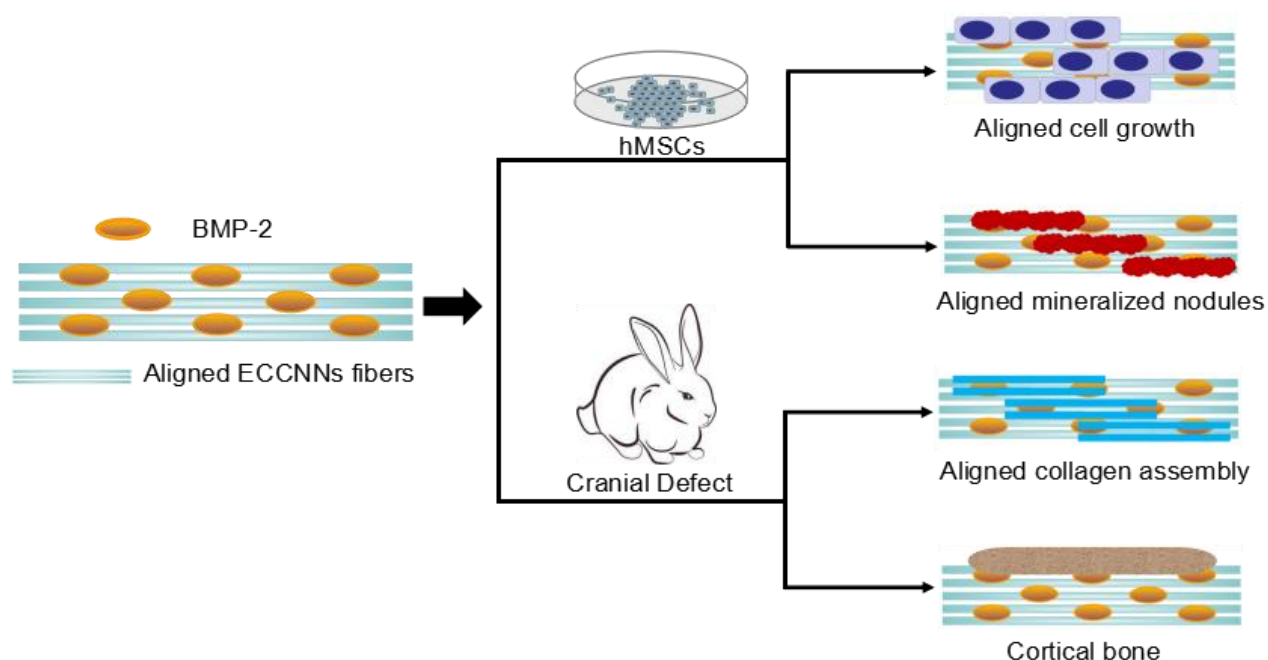

Figure 1. Schematic diagram demonstrating the main experimental procedure in this study

The main study procedure is presents in Figure 1. In this study, aligned and random/unaligned electrospun cellulose nanofibers, enhanced with $20 \%$ CNCs were fabricated (He et al., 2014) and loaded with BMP-2 (see Methods Section for further details).

\subsection{Characterization of ECCNNs loaded with BMP-2.}

The electrospinning process and the obtained cellulose scaffold were demonstrated in Figure 2.a and $b$. The electrospun nanofiber scaffold appeared in white color and had a relatively smooth surface. Figure 2.c, and d presented the SEM images for the random and aligned nanofibers, respectively. SEM images clearly showed a difference in morphology for the two types of nanofibers. Collecting the fibers at a lower rotation speed $(50 \mathrm{~m} / \mathrm{min})$ resulted in random nanofibers, whereas an irregular nonwoven architecture was revealed in the random sample (Figure 2.c), In contrast, a faster rotation speed of the collector $(300 \mathrm{~m} / \mathrm{min}$ ) resulted in stretching of the fibers in the rotating direction of the collector, which gave highly oriented and ordered distribution of individual fibers (Figure 2.d). This finding was consistent with previous studies (De Silva et al., 2018; He et al., 2014; Im, Jung, Jang \& Kim, 2016).

In our previous study, tensile properties of ECCNNs with a range of CNCs contents were measured in directions both along and perpendicular to the fiber orientation (He et al., 2014). In this study, 20 wt.\% CNCs concentration was selected, which is the maximum concentration for electrospinning. The average diameter of nanofibers was $272.4 \pm 31.64 \mathrm{~nm}$ (Figure 2.e) and there was no significant difference in fiber diameter between aligned and random samples. The specific surface area and pore volume of the scaffold were determined by nitrogen adsorption and calculated with BET and BJH methods, respectively. Due to the nano-dimension of ECCNNs, the scaffold exhibited a high surface area of $12 \mathrm{~m}^{2} / \mathrm{g}$ and a pore volume of $0.07 \mathrm{~cm}^{3} / \mathrm{g}$ with a porosity of about 77\% (Figure S1 in Supporting Information).

The total weight of BMP-2 loaded on a $100 \mathrm{~mm}^{2}$ scaffold was $100 \mu \mathrm{g}$. After 7 days BMP-2 
releasing test, the total released BMP-2 was detected to be $0.74 \pm 0.16$ and $0.76 \pm 0.17$ $\mu \mathrm{g} / \mathrm{mm}^{2}$ for aligned and random scaffolds, respectively (Figure 2.f). The relative amount of BMP-2 release (\%) analyzed by ELISA was shown in Figure 2.g. In view of the area of each scaffold $\left(25 \mathrm{~mm}^{2}\right)$, the total incorporated amount of rhBMP-2 in each $5 \times 5 \mathrm{~mm} 2$ sample was $25 \mu \mathrm{g}$. Most BMP-2 was released in the first 5 days. On day 7, the new released BMP-2 was hardly detected. (Figure 2.g)
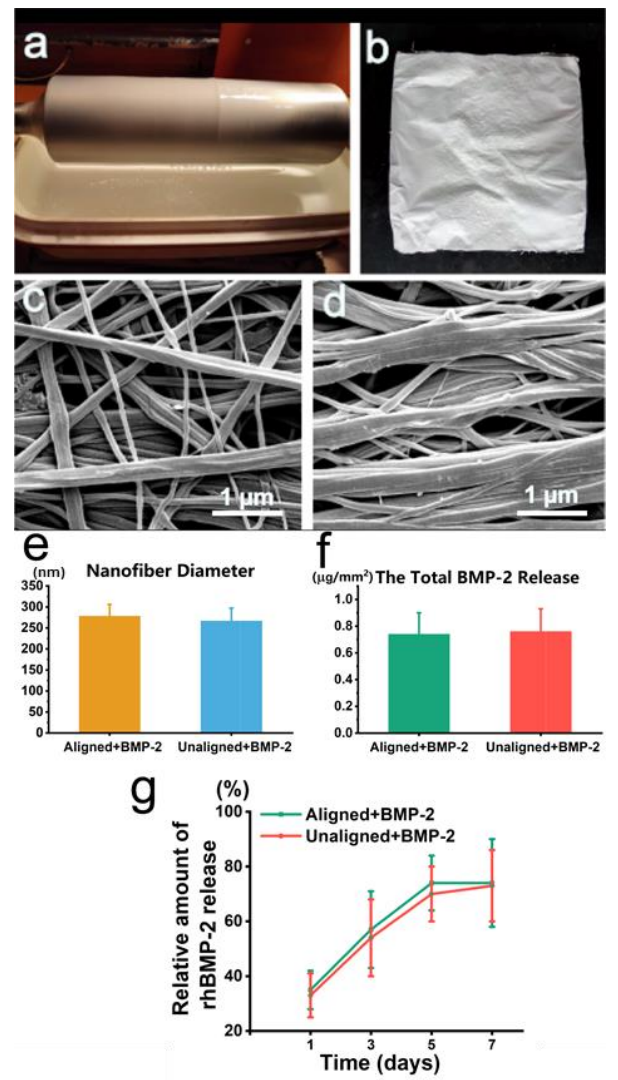

Figure 2. Photos of (a) electrospinning process and (b) obtained scaffold. SEM images of (c) Aligned and (d) Random nanofibers. (e) Average diameter of BMP-2 coated nanofibers. (f) The cumulative amount of BMP-2 released from cellulose electrospun nanofibers in 1 week in vitro. (g) The relative amount rhBMP-2 release compared with the total loaded rhBMP-2. The concentration of BMP-2 was determined by ELISA. Data are expressed as mean \pm standard deviation (SD) of three samples per group.

\subsection{The biocompatibility analysis of scaffolds}

To evaluate the applicability of ECCNNs loaded with BMP-2 for bone regeneration in vitro and in vivo, five groups were set in this study. Scaffolds in the five groups was shown in Table 1.

Table 1. Five different groups compared in this study.

\begin{tabular}{|c|c|c|c|c|c|}
\hline & Group I & Group II & Group III & Group IV & Group V \\
\hline Scaffolds & Aligned+BMP-2 & Aligned & Random+BMP-2 & Random & Empty Control \\
\hline
\end{tabular}




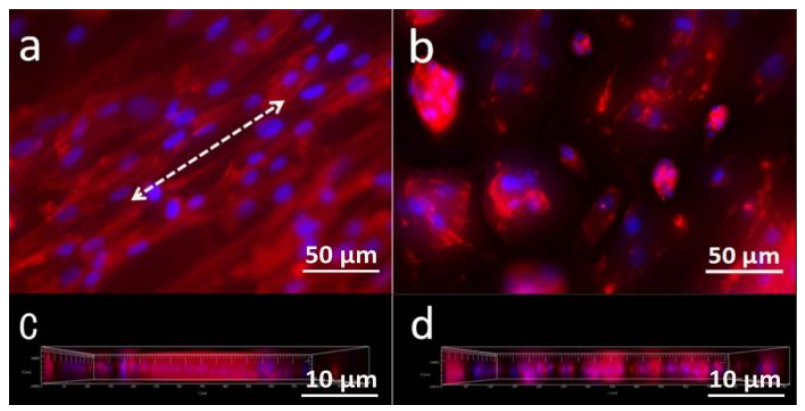

Figure 3. Adhesion and migration of BMSCs in nanofibers. Fluorescence images stained for F-actin (red) and nuclei (blue) of BMSCs on (a) Aligned and (b) Random nanofibers after $72 \mathrm{~h}$ of culture. After 3D reconstruction of CLSM images, cells could be immersed into (c) Aligned and (d) Random nanofibers after $72 \mathrm{~h}$ of culture.

BMSCs were isolated and cultured for biocompatibility evaluation. Cell proliferation in the five groups were investigated by MTS. The proliferation of BMSCs cultured on scaffolds exhibited no significant difference between each group and empty control on day 1,3,5,7 (Figure.S1). It suggested all the scaffolds possess great biological compatibility and are suitable for BMSCs growth and proliferation.

BMSCs growth situation on scaffolds were analyzed by laser scanning microscopy (CLSM). CLSM images showed sufficient cell adhesion and widely spread cell morphology on both aligned (Figure 3.a) and random scaffolds (Figure 3.b) with obvious difference when observed by CLSM. On aligned scaffold, the cells had spindle shape morphology and stretched along the long axis of the underlying nanofibers (The direction of white line with double-arrow heads). On random scaffold, BMSCs were more polygonal in shape and distributed randomly throughout the nanofibers. After 72 hours of culture, the cells seeded onto the scaffolds surface migrated to the full depth of the aligned (Figure 3.c) and random (Figure 3.d) scaffold (depth=150 $\mu \mathrm{m}$ ). These results suggested that aligned and random scaffolds have obvious BMSCs guiding potential. The change in cell adhesion and elongated cellular morphology on nanofibrous scaffolds can be explained by the classical theory of contact guidance (Jamaiyar et al., 2017). Aligned nanofibers have recently been explored to employ spatial instruction for different stem cells types, such as endothelial cells (Chou et al., 2017), neuronal cells (Hyysalo, Ristola, Joki, Honkanen, Vippola \& Narkilahti, 2017), cardiomyocytes (Wanjare, Hou, Nakayama, Kim, Mezak \& Abilez, 2017), and BMSCs (Perikamana et al., 2015). When BMSCs were cultured on aligned poly(L-lactic acid) (PLLA) nanofibers, the same morphological changes were observed as we reported (Perikamana et al., 2015).

Excellent BMSCs adhesion, proliferation and migration on ECCNNs suggest that the hydrophilic and nanostructured fibers facilitated cell-scaffold interactions. This could be through improved receptor recognition of fibrillary fibrin, or by increased surface area and roughness, which have been reported to enhance osteoblasts proliferation and differentiation (Saranya, Saravanan, Moorthi, Ramyakrishna \& Selvamurugan, 2011). 


\subsection{Mineralization capacity test in vitro}

Mineralization of BMSCs cultured in osteogenic differentiation medium on different scaffolds was examined using ALP activity and calcium content measurement in each group on day 14 and 21 (Figure 4.a and b). On day 14, ALP activity in the Aligned+ BMP-2 group (6.141 \pm 2.503 ) and Random + BMP-2 group $(6.362 \pm 2.820)$ were significantly higher than that in empty control group $(1.642 \pm 0.835, \mathrm{P}<0.05)$. The difference between empty control and random scaffold group had no significance; On day 21, ALP activity in Aligned+ BMP-2 group (10.894 \pm 3.403$)$, Aligned group (4.168 \pm 2.462$)$, Random + BMP-2 group (9.678 \pm 4.276 ) and Random group (4.915 \pm 1.279$)$ were significantly higher than that in empty control group $(1.221 \pm 0.657, \mathrm{P}<0.01)$. Adding BMP-2 could significantly increase the ALP activity, no matter in Aligned or Random scaffolds (Figure 4.a).

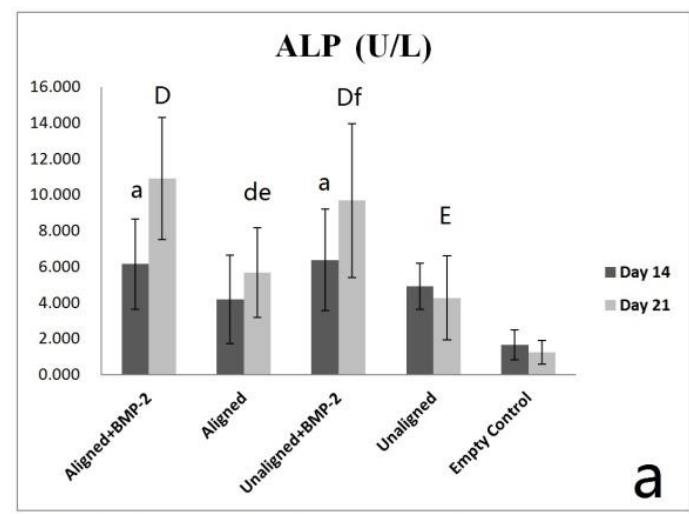

a $P<0.05$ compared to control group on Day 14 . ${ }^{\mathrm{b}} P<0.05$ compared Aligned+BMP2 group on Day 14 ${ }^{c} P<0.05$ compared to Unaligned group on Day 14 . d $P<0.05$ compared to control group on Day 21 . e $P<0.05$ compared to Aligned+BMP2 group on Day 21 . ${ }^{\mathrm{f}} P<0.05$ compared to Unaligned group on Day 21 . g $P<0.05$ compared to Aligned group on Day 14 .

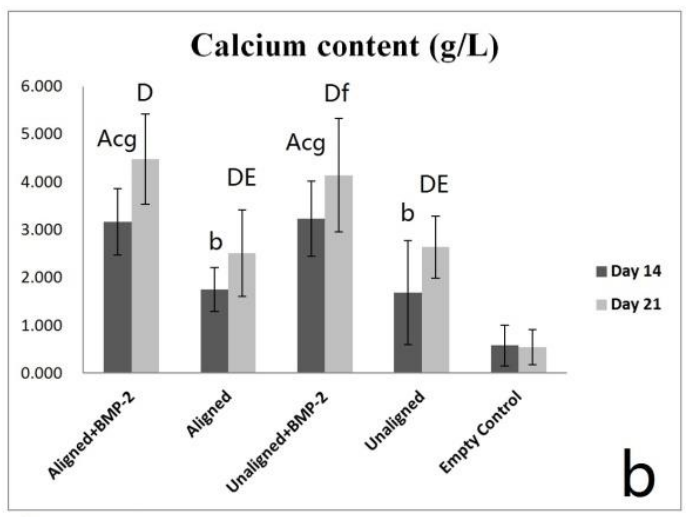

A $P<0.01$ compared to control group on Day 14 .

${ }^{\mathrm{B}} P<0.01$ compared to Aligned+BMP2 group on Day 14

${ }^{\mathrm{C}} P<0.01$ compared to Unaligned group on Day 14 . $\mathrm{D} P<0.01$ compared to control group on Day 21 .

${ }^{\mathrm{E}} P<0.01$ compared to Aligned+BMP2 group on Day 21 .

${ }^{\mathrm{F}} P<0.01$ compared to Unaligned group on Day 21 .

${ }^{\mathrm{G}} P<0.01$ compared to Aligned group on Day 14 .

Figure 4. (a) ALP activities and (b) Calcium content of BMSCS cultured on five different groups in osteogenic differentiation on day 14 and 21 . DMEM $+10 \%$ fetal calf serum and culture plate was used as empty control. Data are average \pm standard deviation from 3 samples.

On day 14 , calcium content in the Aligned + BMP-2 group ( $3.16 \pm 0.696)$ and Random + BMP-2 group (3.228 \pm 0.785 ) were significantly higher than those in empty control group $(0.574 \pm 0.423$, $\mathrm{P}<0.01)$, Aligned group $(1.747 \pm 0.464, \mathrm{P}<0.05)$ and Random group $(1.685 \pm 1.088, \mathrm{P}<0.05)$. The difference between aligned and random scaffold group with BMP-2 had no significance; On day 21, calcium content in Aligned+ BMP-2 group (4.477 \pm 0.947$)$, Aligned group ( $2.509 \pm 0.909)$, Random + BMP-2 group $(4.139 \pm 1.19)$ and Random group $(2.632 \pm 0.648)$ were significantly higher than that in empty control group $(0.541 \pm 0.373, \mathrm{P}<0.01)$. Adding BMP-2 could significantly increase the calcium content, no matter in Aligned or Random scaffolds (Figure 4.b). The ALP and calcium content tests showed that similar levels of osteogenic differentiation of BMSCs could be observed in BMP-2 loading or non-loading groups, irrespective of fiber morphology as well as cell morphology. 
After 28 days osteogenic differentiation, alizarin red S staining was done (Figure 5.). In contrast, no staining was observed in empty group. From scaffold with BMP-2 group (Figure 5.a, and 5.c), Alizarin red S staining was stronger than scaffold without BMP-2 groups (Figure 5.b, and 5.d). Organized Alizarin red S staining calcium nodules could be seen in aligned scaffold groups (Figure 5.a, and 5.b). Random scaffold groups just showed random alizarin red $\mathrm{S}$ staining calcium nodules (Figure 5.c, and 5.d).

Alizarin red $\mathrm{S}$ staining images showing well-organized mineralized nodules assembly were observed after 28 days of culture in an osteogenic medium on aligned ECCNNs. Loading BMP-2 increased the quantity of calcium nodules, which followed the same trend of initial cell adhesion. It is conceivable that cells may prefer to continue their initial cell adhesion behavior and that ECM secretion can also follow the same pattern, which is consistent with other studies (Liu, Wei, Zhang, Xu, Yang \& Deng, 2013).

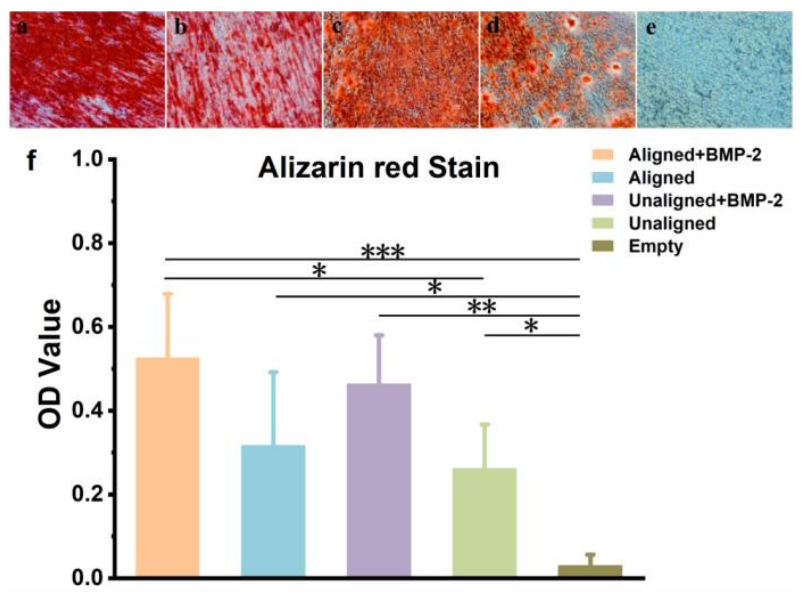

Figure 5. Alizarin red S staining of BMSCs cultured in osteogenic medium for 28 days on different scaffolds. (a) Aligned+BMP-2 group, (b) Aligned group, (c) Random+BMP-2 group, (d) Random group, (e) Empty Control group, (f) Plotted graph represents an Alizarin red Stain based assay of mineralization. Using culture plate with DMEM and $10 \%$ fetal as empty control. $\left(^{*}, \mathrm{P}<0.05\right.$, compared with a line connecting two groups; ${ }^{*}, \mathrm{P}<0.01$, compared with a line connecting two groups).

In general, different physical factors such as surface topography, stiffness, and shear stress are influential in osteogenic differentiation of different cells, though osteogenic differentiation on aligned and random nanofibers is slightly controversial (Perikamana et al., 2015). For example, Liu et al. claimed that bone marrow mesenchymal stem cells (BMSCs) cultured on random nanofibers showed increased ALP activity and higher expression of osteogenic genes as compared to an aligned substrate (Lyu, Huang, Yang \& Zhang, 2013). Contrary to these results, Perikamana.et al (Perikamana et al., 2015) demonstrated that aligned nanofibers showed higher osteogenic differentiation properties than random nanofiber groups did (increase ALP activity and calcium content). Similarly, higher expression of osteogenic gene markers such as RUNX ${ }_{2}$, OSX, COL-1, and OCN were observed on aligned nanofibers (Scaglione et al., 2012). 


\subsection{Bone regeneration test in vivo.}

In order to demonstrate the bone regeneration ability of this scaffold in vivo, rabbit cranial defect model was built. Scaffolds were implanted into calvaria bone defect area (Figure 6). The postoperative course of the 12 rabbits was uneventful during the healing period. The scaffolds exhibited good tissue compatibility and no adverse events such as foreign body reaction or infection were observed.
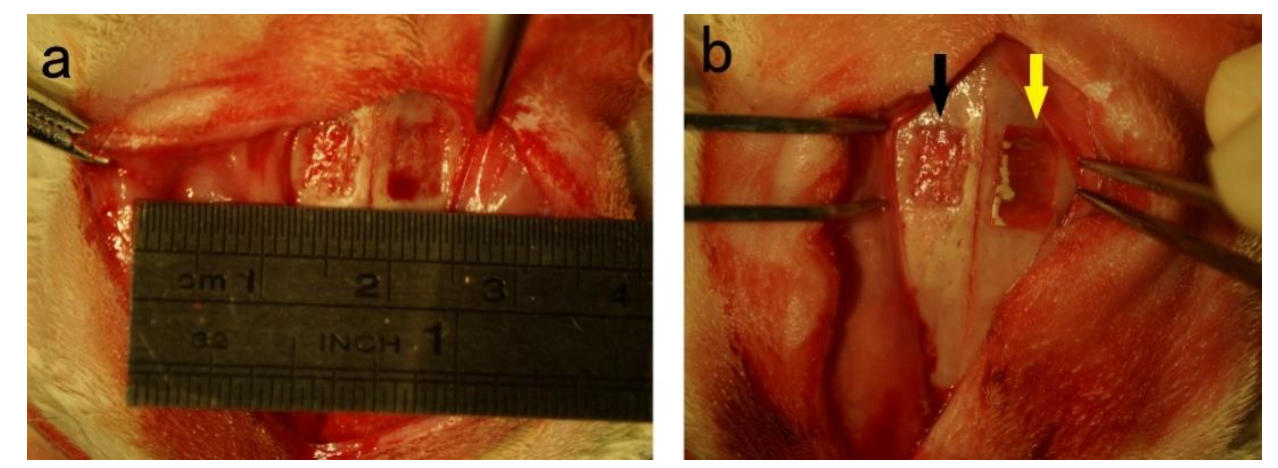

Figure 6. Intraoperative image, $5 \times 5 \mathrm{~mm}$ square defect was made on each side of rabbit calvaria bone, (a) empty bone defect area (Black arrow) and (b) bone defect area implanted with scaffold (Yellow arrow).

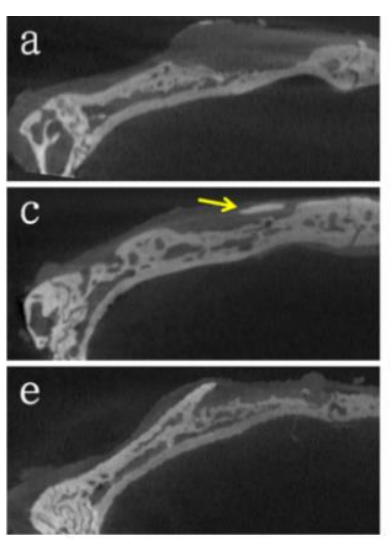

$\mathrm{g}$

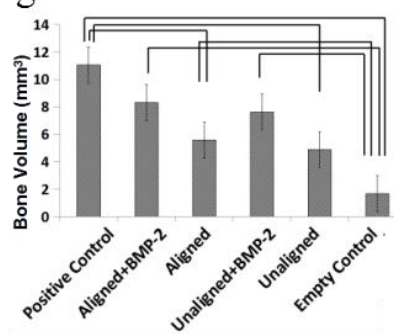

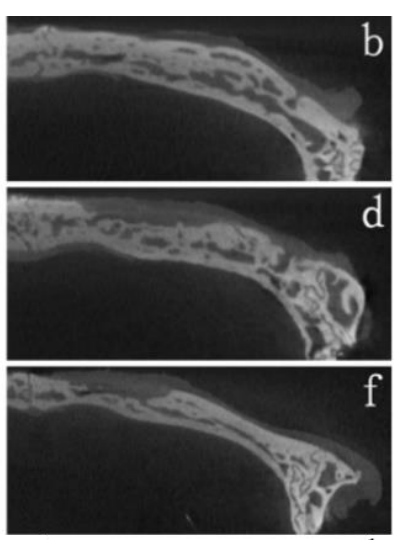

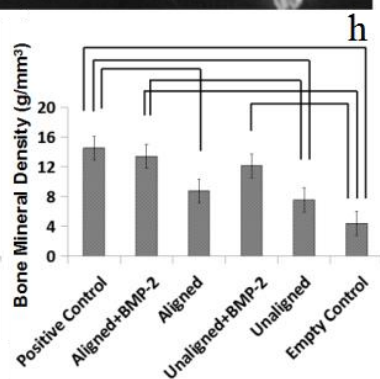

Figure 7. Micro CT images of representative samples from 6 different groups. (a) Negative control, (b) Positive control, (c) Aligned scaffold with BMP-2, (d) Random scaffold with BMP-2 (e) Aligned scaffold without BMP-2, (f) Random scaffold without BMP-2 (Scale bar: $2 \mathrm{~mm}$ ), (g) Bone volume and (h) Bone mineral density was quantitatively measured. The difference is significant $(P<0.05)$ when compared between two groups with a connecting line. 
Micro CT analysis was carried out for structural and quantitative assessments of new bone formation and mineralization in the defective site. Two-dimensional (2D) micro-CT images showed the morphology of cranial defect area. After 12 weeks of surgery, negative control (Figure 7.a) is almost still empty, little new bone formed at the defect site margin. Positive control (Figure 7.b) is normal cranial micro CT image without surgery. Aligned (Figure 7.c) and random (Figure 7.d) electrospun cellulose scaffolds loaded with BMP-2 were significantly different from the negative control. More spongy bone was found from the endocardium side, comparing with the without BMP-2 groups, neither aligned (Figure 7.e) nor random (Figure 7.f) had much bone formation. Remarkably, some cortical bone could be seen on the skin side in aligned with BMP-2 group, even it had not shown integrity in the defect side (the yellow arrow in Figure 7.c).

Furthermore, the volume of the newly formed bone volume (BV) and bone mineral density (BMD), the most important parameters of new bone regeneration, were evaluated (Figure 7.g-h). The values of BV and BMD in aligned group $\left(8.63 \mathrm{~mm}^{3}, 14.09 \mathrm{~g} / \mathrm{cm}^{3}\right)$ and random group $(7.62$ $\mathrm{mm}^{3}, 12.15 \mathrm{~g} / \mathrm{cm}^{3}$ ) loaded with BMP-2 were significantly higher as compared to those for the defect-only group $\left(1.69 \mathrm{~mm}^{3}, 4.42 \mathrm{~g} / \mathrm{cm}^{3}, \mathrm{P}<0.001\right)$, and the difference was not significant when compared with positive control group $\left(10.68 \mathrm{~mm}^{3}, 15.01 \mathrm{~g} / \mathrm{cm}^{3}, \mathrm{P}>0.05\right)$. The BV of aligned group $\left(5.58 \mathrm{~mm}^{3}\right)$ is also significantly higher than that of defect-only group $(P<0.05)$, while no significant difference was observed in BMD of aligned group $\left.\left(8.12 \mathrm{~g} / \mathrm{cm}^{3}, \mathrm{P}<0.05\right)\right)$, BV and BMD of unaligned group $\left(5.22 \mathrm{~mm}^{3}, 7.64 \mathrm{~g} / \mathrm{cm}^{3}\right)$ when comparing to the defect-only group $\left.(P<0.05)\right)$. Furthermore, both BV and BMD were significant lower in aligned and random groups without BMP-2 loading when compared with positive control group $(P<0.05)$. It means aligned and unaligned/random scaffolds could induce bone formation in vivo and especially increase the BV parameter. In addition, loading BMP-2 on ECCNNs could significantly enhance bone formation.

Masson trichrome staining demonstrated new bone formation and a distribution of regenerated collagen fibers throughout the bone defect region (Figure 8.a-f). In the aligned ECCNNs with rhBMP-2 group, the nanofibers were completely covered by newly formed aligned collagen fibers, which were integrated into the host bone tissues (Figure 8.g). In random ECCNNs with rhBMP-2 group, the new collagen fiber is also random (Figure 8.f).

The orientation of the cells was further confirmed by H\&E images (Data was shown as supporting information). In H\&E staining images, cell nucleus was stained blue and cytoplasm was red. In random group, the cell nucleus and the cytoplasm lay randomly; In aligned group, both the cell nucleus and the cytoplasm followed the same direction (yellow double arrow line), as parallel to the orientation direction of the nanofibers in scaffold (Figure.S2a). In contrast, the cells and cell nucleus around the random ECCNNs with BMP-2 scaffold (Figure.S2b) were distributed randomly, which confirmed the irregular orientation of the nanofibers. Cells cultured on ECCNNs without BMP-2 have similar image just with less bone formation (data not shown). 


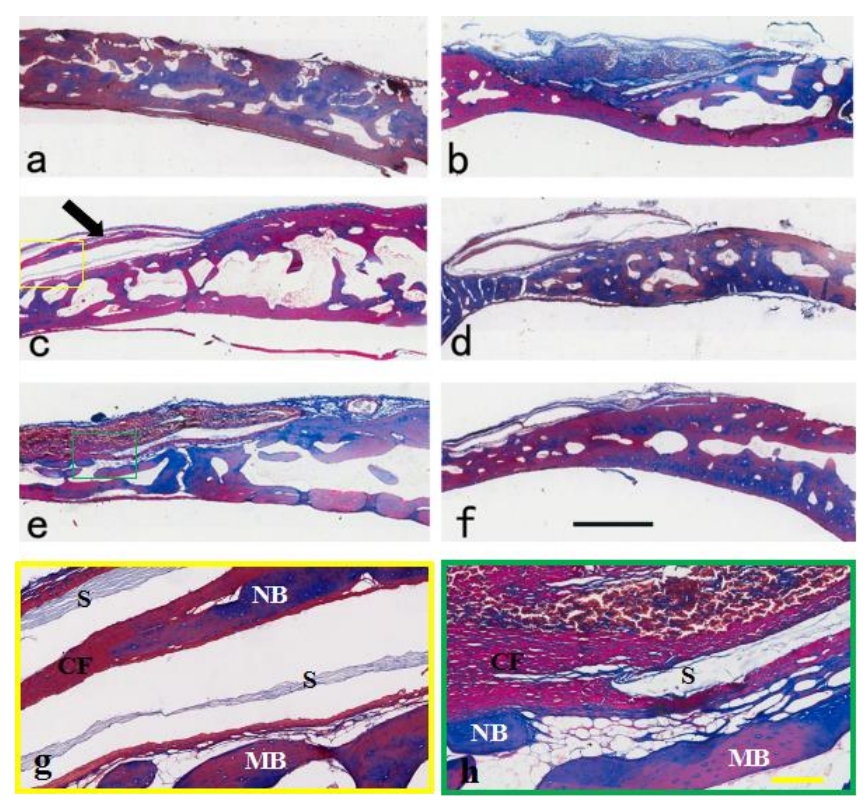

Figure 8. Masson's Trichrome Stain of representative samples from 6 groups. In these images the red stain represents osteoid and blue stain indicates new bone formation (Masson's trichrome stain). (a) Positive control, (b) Negative control, (c) aligned scaffold with BMP-2 group, (d) aligned scaffold without BMP-2 group, (e) Random scaffold with BMP-2 group, (f) random scaffold without BMP-2 group (Scale bar: $2 \mathrm{~mm}$ ), (g) shows magnified aligned collagen fibers of yellow rectangle in (c), (h) shows magnified area of random collagen fibers in green rectangle of (e). Labels indicate: Scaffold (S), New bone (NB), Mature bone (MB) and Collagen fibers (CF) (Scale bar: $400 \mu \mathrm{m}$ ).

Under Masson's trichrome staining, old bone is stained red, new bone stained in blue and collagen fiber stained in red. The positive control (Figure 8.a) is normal cranial sample without surgery. The negative control (Figure 8.b) is almost still empty, little new bone formatted at the defect site margin. Some new sponge bone had regenerated in aligned (Figure 8.c) and random (Figure 8.d) electrospun cellulose scaffolds loaded with BMP-2 groups. Even in the aligned (Figure 8.e) and random (Figure 8.f) without BMP-2 groups, some new sponge bone has been regenerated at the endocardium side. Among these 6 groups, only in ECCNNs with BMP-2 group, cortical bone could be seen above the scaffold (Figure 8.c Black arrow).

Bone is a highly dynamic tissue that is continuously remodeled during its normal growth or following injury. Woven bone is initially produced during bone remodeling and is characterized by a mechanically weak, disorganized arrangement of collagen fibers. However, during the bone remodeling process, woven bone is eventually replaced by regular, parallel aligned collagen fibers that form a sheet-like structure called lamellar bone. There is recent evidence that the structure of the implanted biomaterials can influence the collagen deposition pattern (Perikamana et al., 2015). In this study, we observed that collagen remodeling was strictly controlled by the nanofiber architecture. A lamellar-like collagen assembly was found on aligned nanofibers, whereas a disorganized, woven-like pattern was observed on the random fibers during the same time period. Under natural conditions, bone remodeling is a function of the response of the bone to external mechanical loading (Wolf's law) (Molster, Gjerdet, Alho \& Bang, 1983). Besides, for bone tissue engineering, a stronger mechanical strength of the scaffold was needed for the cortical bone than sponge bone in vivo (Roohani-Esfahani, Newman \& Zreiqat, 2016). Though cellulose has the most abundant nature source with excellent 
biocompatibility, electrospun cellulose nanofibers were rarely studied in bone tissue engineering. In our study, aligned ECCNNs could induce cell growth orderly, mineralize calcium nodules orderly in vitro, and secret collagen orderly in vivo, which is similar to the aligned electrospun poly(L-lactic acid) (PLLA) nanofibers in bone regeneration study (Perikamana et al., 2015). Moreover, aligned ECCNNs exhibited a much stronger mechanical strength than normal electrospun cellulose nanofibers. This may be the reason that cortical bone could be seen above the scaffold of aligned ECCNNs loaded with BMP-2, suggesting that the mechanically strengthened and aligned cellulose nanofibers could influence collagen remodeling into cortical bone during bone healing. Comparing with a similar study that aligned collagen was found in aligned electrospun PLLA nanofibers, however, no obvious cortical bone could be detected. This should be possibly ascribed to the fact that the PLLA scaffold is much weaker in mechanical strength than aligned ECCNNs scaffold (Perikamana et al., 2015).

Besides, for tissue engineering applications, scaffolds should have well-controlled pore size and interconnections (Soliman, Sant, Nichol, Khabiry, Traversa \& Khademhosseini, 2011). Increasing porosity results in greater bone ingrowth and enhanced bone formation, leading to direct osteogenesis without osteochondral formation as reported by others (Sadowska, Wei, Guo, Guillem-Marti, Ginebra \& Xiao, 2018). However, pore sizes larger than $500 \mathrm{~nm}$ are associated with fibrovascular tissue formation (Smyth, Fournier, Driemeier, Picart, Foster \& Bras, 2017; van Tienen, Heijkants, Buma, de Groot, Pennings \& Veth, 2002). The pore size of ECCNNs is about $100-600 \mathrm{~nm}$ with a porosity of $77 \%$, which is similar to bone matrix structure (Murphy, O'Brien, Little \& Schindeler, 2013; Wu et al., 2018). The interconnected pore structure of the ECCNNs scaffolds assists both osteoblasts ingrowth and distribution within the scaffolds and thus may facilitate angiogenesis and diffusion of nutrients. In addition, the average fiber diameter in each group was at 250-350 $\mathrm{nm}$ (Figure.2e) and is similar to that of collagen fibers in the bone extracellular microenvironment, which may recruit and induce more endogenous stem cells from endocranium (He et al., 2014) even without BMP-2 loading. Besides, the nanofiber scaffold transfers $2 \mathrm{D}$ cell culture into $3 \mathrm{D}$ culture, more spaces are available to host cell growth as compared with the culture plate in vitro (Duval et al., 2017). For aligned nanofiber scaffold, the aligned cell growth pattern makes the cell contact inhibition further reduced (Figure 7.g). (Nuhn, Perez \& Schneider, 2018).

Finally, we propose a possible mechanism of in vivo collagen matrix assembly guided by aligned ECCNNs with BMP-2, which involved 5 aspects: 1). Electrospun nanofiber scaffold has a similar structure to ECM and cellulose is biocompatible, which could induce the "homing effect" of stem cells and support their attachment; 2) Through contact guidance, aligned nanofibers instructed endogenous progenitor cells adhesion and growth by the nanofiber morphology; 3 ) Loading BMP-2 on scaffold actively turns endogenous progenitor cells into osteoblasts; 4) The osteoblasts secrete collagen along the direction of cell adhesion (fiber direction), which subsequently forms the collagen fibril bundles in the direction of deposited collagen molecules; 5) Individual collagen fibrils may undergo directional mineralization, and these mineralized structures are then assembled together to form an organized structure of bone (cortical bone).

\section{CONCLUSION}

The results of the present study suggest that the combination of BMP-2 and aligned ECCNNs scaffold has great potential for bone regeneration. Aligned cellulose nanofibers could induce aligned $\mathrm{BMSC}$ growth and mineralized nodules formation in vitro, and assembly aligned 
600

601

602

603

604

605

606

607

608

609

610

611

612

613

614

615

616

617

618

619

620

621

622

623

collagen and cortical bone formation in vivo. The study provides a basis for future optimization of electrospun nanofibrous scaffolds for bone tissue engineering applications.

\section{ASSOCIATED CONTENT}

Supporting Information

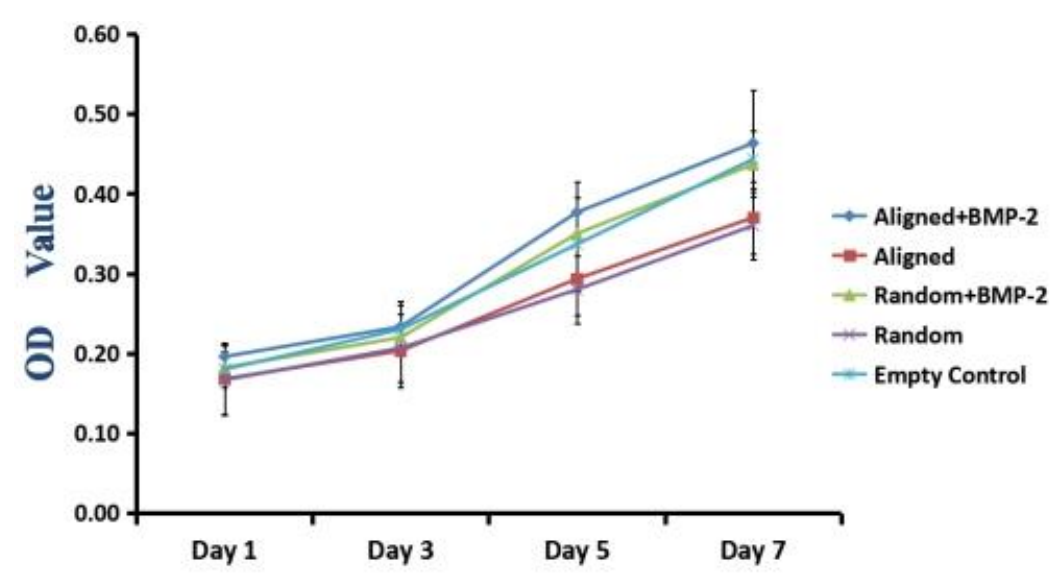

Figure.S1 Analysis of the proliferation of BMSCS on different group by MTS assay on day 1, 3, 5, 7. Bars represent the means $\pm \mathrm{Sd}$. There was no significant difference between different groups at each day. Plastic culture plate was used as Empty Control.
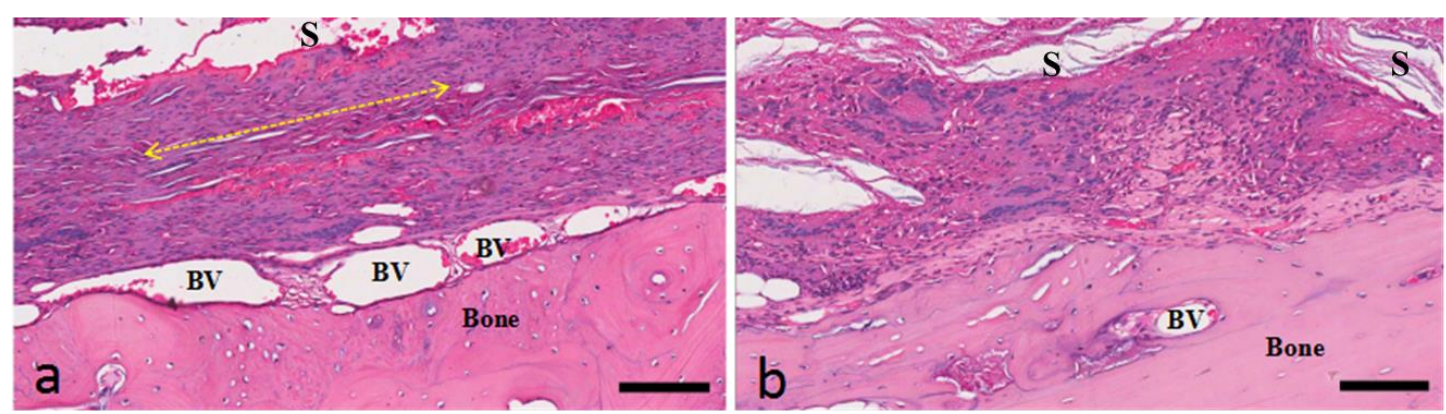

Figure.S2. H\&E Stain. Aligned and random cells and collagen could be seen in aligned (a) and random (b) groups. Aligned cellulose Loading BMP-2 gave more new bone formation. BV: Blood vessel, S: scaffold.

\section{ACKNOWLEDGEMENT}

This work was supported by Natural Science Foundation of China (NSFC) grants (31500789, 51433006, 51473100, 81870758 and 31871464), Chongqing Yuzhong District science and technology plan project grants (20170124), Chongqing Research Program of Basic Research and Frontier Technology (cstc2018jcyjAX0807, cstc2017jcyjBX0019 and cstc2017jcyjAX0020), Temple University Kornberg School of Dentistry research start-up funds, the RCUK China-UK Science Bridges Program through the Medical Research Council and the Engineering and 
Physical Sciences Research Council and Program for Innovation Team 1015 Building at Institutions of Higher Education (No. 1016 CXTDG201602006) funded by the Chongqing Municipal 1017 Education Commission of China in 2016.

\section{The authors declare no competing financial interest.}

\section{References:}

Amini, N., Vousooghi, N., Alizade, A., Ramezani, S., Joghataei, M. T., Milan, P. B., Mehrabi, S., Ababzadeh, S., Sefat, F., \& Mozafari, M. (2018). Transplantation of adipose tissue-derived stem cells into brain through cerebrospinal fluid in rat models: Protocol development and initial outcome data. Curr Stem Cell Res Ther.

Bertrand, N., Grenier, P., \& Mahmoudi, M. (2017). Mechanistic understanding of in vivo protein corona formation on polymeric nanoparticles and impact on pharmacokinetics. 8(1), 777.

Chou, C. L., Rivera, A. L., Williams, V., Welter, J. F., Mansour, J. M., Drazba, J. A., Sakai, T., \& Baskaran, H. (2017). Micrometer scale guidance of mesenchymal stem cells to form structurally oriented largescale tissue engineered cartilage. Acta Biomater, 60, 210-219.

Dadsetan, M., Guda, T., Runge, M. B., Mijares, D., LeGeros, R. Z., LeGeros, J. P., Silliman, D. T., Lu, L., Wenke, J. C., Brown Baer, P. R., \& Yaszemski, M. J. (2015). Effect of calcium phosphate coating and rhBMP-2 on bone regeneration in rabbit calvaria using poly(propylene fumarate) scaffolds. Acta Biomater, 18, 9-20.

Daghigh Ahmadi, E., Israr Raja, T., Ali Khaghani, S., Fhong Soon, C., Mozafari, M., Youseffi, M., \& Sefat, F. (2018). The role of photonics and natural curing agents of TGF- $\beta 1$ in treatment of osteoarthritis. Materials Today: Proceedings, 5(7), 15540-15549.

De Silva, R. T., Dissanayake, R. K., Mantilaka, M., Wijesinghe, W., Kaleel, S. S., Premachandra, T. N., Weerasinghe, L., Amaratunga, G. A. J., \& de Silva, K. M. N. (2018). Drug-Loaded Halloysite NanotubeReinforced Electrospun Alginate-Based Nanofibrous Scaffolds with Sustained Antimicrobial Protection.

Deshpande, P., Ramachandran, C., Sefat, F., Mariappan, I., Johnson, C., McKean, R., Hannah, M., Sangwan, V. S., Claeyssens, F., Ryan, A. J., \& MacNeil, S. (2013). Simplifying corneal surface regeneration using a biodegradable synthetic membrane and limbal tissue explants. Biomaterials, 34(21), 5088-5106. 
Driscoll, M. K., Sun, X., Guven, C., Fourkas, J. T., \& Losert, W. (2014). Cellular contact guidance through dynamic sensing of nanotopography. ACS Nano, 8(4), 3546-3555.

Du, J., \& Hsieh, Y.-L. (2009). Cellulose/chitosan hybrid nanofibers from electrospinning of their ester derivatives.

Duval, K., Grover, H., Han, L. H., Mou, Y., Pegoraro, A. F., Fredberg, J., \& Chen, Z. (2017). Modeling Physiological Events in 2D vs. 3D Cell Culture. 32(4), 266-277.

Filion, T. M., Skelly, J. D., Huang, H., Greiner, D. L., Ayers, D. C., \& Song, J. (2017). Impaired osteogenesis of T1DM bone marrow-derived stromal cells and periosteum-derived cells and their differential in-vitro responses to growth factor rescue. Stem Cell Res Ther, 8(1), 65.

Frey, M. (2008). Electrospinning Cellulose and Cellulose Derivatives.

He, X., Xiao, Q., Lu, C., Wang, Y., Zhang, X., Zhao, J., Zhang, W., Zhang, X., \& Deng, Y. (2014). Uniaxially aligned electrospun all-cellulose nanocomposite nanofibers reinforced with cellulose nanocrystals: scaffold for tissue engineering. Biomacromolecules, 15(2), 618-627.

Hyysalo, A., Ristola, M., Joki, T., Honkanen, M., Vippola, M., \& Narkilahti, S. (2017). Aligned Poly(epsilon-caprolactone) Nanofibers Guide the Orientation and Migration of Human Pluripotent Stem Cell-Derived Neurons, Astrocytes, and Oligodendrocyte Precursor Cells In Vitro. Macromol Biosci, 17(7).

Im, S. H., Jung, Y., Jang, Y., \& Kim, S. H. (2016). Poly(L-lactic acid) scaffold with oriented micro-valley surface and superior properties fabricated by solid-state drawing for blood-contact biomaterials. Biofabrication, 8(4), 045010.

J. Bye, F., Bullock, A., Singh, R., Sefat, F., Roman, S., \& Macneil, S. (2014). Development of a Basement Membrane Substitute Incorporated Into an Electrospun Scaffold for 3D Skin Tissue Engineering.

Jamaiyar, A., Wan, W., Ohanyan, V., Enrick, M., Janota, D., Cumpston, D., Song, H., Stevanov, K., Kolz, C. L., Hakobyan, T., Dong, F., Newby, B. Z., Chilian, W. M., \& Yin, L. (2017). Alignment of inducible vascular progenitor cells on a micro-bundle scaffold improves cardiac repair following myocardial infarction. Basic Res Cardiol, 112(4), 41.

Jayaramudu, J., Reddy, G. S., Varaprasad, K., Sadiku, E. R., Sinha Ray, S., \& Varada Rajulu, A. (2013). Preparation and properties of biodegradable films from Sterculia urens short fiber/cellulose green composites. Carbohydr Polym, 93(2), 622-627.

Kishan, A. P., \& Cosgriff-Hernandez, E. M. (2017). Recent advancements in electrospinning design for 
Liu, W., Wei, Y., Zhang, X., Xu, M., Yang, X., \& Deng, X. (2013). Lower extent but similar rhythm of osteogenic behavior in hBMSCs cultured on nanofibrous scaffolds versus induced with osteogenic supplement. ACS Nano, 7(8), 6928-6938.

Lyu, S., Huang, C., Yang, H., \& Zhang, X. (2013). Electrospun fibers as a scaffolding platform for bone tissue repair. J Orthop Res, 31(9), 1382-1389.

Ma, M. G., Qing, S. J., Li, S. M., Zhu, J. F., Fu, L. H., \& Sun, R. C. (2013). Microwave synthesis of cellulose/CuO nanocomposites in ionic liquid and its thermal transformation to CuO. Carbohydr Polym, 91(1), 162-168.

Magalhaes, W. L., Cao, X., \& Lucia, L. A. (2009). Cellulose nanocrystals/cellulose core-in-shell nanocomposite assemblies. Langmuir, 25(22), 13250-13257.

Mahjour, S. B., Fu, X., Yang, X., Fong, J., Sefat, F., \& Wang, H. (2015). Rapid creation of skin substitutes from human skin cells and biomimetic nanofibers for acute full-thickness wound repair. Burns, 41(8), 1764-1774.

Murphy, C. M., O'Brien, F. J., Little, D. G., \& Schindeler, A. (2013). Cell-scaffold interactions in the bone tissue engineering triad. Eur Cell Mater, 26, 120-132.

Mohamadi, F., Ebrahimi-Barough, S., Nourani, M. R., Mansoori, K., Salehi, M., Alizadeh, A. A., Tavangar, S. M., Sefat, F., Sharifi, S., \& Ai, J. (2017). Enhanced sciatic nerve regeneration by human endometrial stem cells in an electrospun poly (epsilon-caprolactone)/collagen/NBG nerve conduit in rat. Artif Cells Nanomed Biotechnol, 1-13.

Mohammadi, A., Maleki-Jamshid, A., Sanooghi, D., Milan, P. B., Rahmani, A., Sefat, F., Shahpasand, K., Soleimani, M., Bakhtiari, M., Belali, R., Faghihi, F., Joghataei, M. T., Perry, G., \& Mozafari, M. (2018). Transplantation of Human Chorion-Derived Cholinergic Progenitor Cells: a Novel Treatment for Neurological Disorders.

Molster, A. O., Gjerdet, N. R., Alho, A., \& Bang, G. (1983). Fracture healing after rigid intramedullary nailing in rats. Acta Orthop Scand, 54(3), 366-373. 
Moztarzadeh, S., Mottaghy, K., Sefat, F., Samadikuchaksaraei, A. and Mozafari, M. (2018) Nanoengineered biomaterials for lung regeneration. Nanoengineered Biomaterials for Regenerative Medicine, pp.305-323.

Nada, A. A., Abdellatif, F. H. H., Ali, E. A., Abdelazeem, R. A., Soliman, A. A. S., \& Abou-Zeid, N. Y. (2018). Cellulose-based click-scaffolds: Synthesis, characterization and biofabrications. Carbohydr Polym, 199, 610-618.

Nejatian, T., Khurshid, Z., Zafar, M. S., Najeeb, S., Zohaib, S., Mazafari, M., Hopkinson, L., \& Sefat, F. (2017). 5 - Dental biocomposites. Biomaterials for Oral \& Dental Tissue Engineering, 65-84.

Nuhn, J. A. M., Perez, A. M., \& Schneider, I. C. (2018). Contact guidance diversity in rotationally aligned collagen matrices. Acta Biomater, 66, 248-257.

O'Donnell, N., Okkelman, I. A., Timashev, P., Gromovykh, T. I., Papkovsky, D. B., \& Dmitriev, R. I. (2018). Cellulose-based scaffolds for fluorescence lifetime imaging-assisted tissue engineering. Acta Biomater.

Ortega, Í., Sefat, F., Deshpande, P., Paterson, T., Ramachandran, C., Ryan, A., MacNeil, S. and Claeyssens, F. (2014) Combination of Microstereolithography and Electrospinning to Produce Membranes Equipped with Niches for Corneal Regeneration. Journal of Visualized Experiments, (91).

Perikamana, S. K., Lee, J., Ahmad, T., Jeong, Y., Kim, D. G., Kim, K., \& Shin, H. (2015). Effects of Immobilized BMP-2 and Nanofiber Morphology on In Vitro Osteogenic Differentiation of hMSCs and In Vivo Collagen Assembly of Regenerated Bone. ACS App/ Mater Interfaces, 7(16), 8798-8808.

Podsiadlo, P., Choi, S. Y., Shim, B., Lee, J., Cuddihy, M., \& Kotov, N. A. (2005). Molecularly engineered nanocomposites: layer-by-layer assembly of cellulose nanocrystals. Biomacromolecules, 6(6), 29142918.

Podsiadlo, P., Sui, L., Elkasabi, Y., Burgardt, P., Lee, J., Miryala, A., Kusumaatmaja, W., Carman, M. R., Shtein, M., Kieffer, J., Lahann, J., \& Kotov, N. A. (2007). Layer-by-layer assembled films of cellulose nanowires with antireflective properties. Langmuir, 23(15), 7901-7906.

Raja, T., Khaghani, S., Zafar, M., Khurshid, Z., Mozafari, M., Youseffi, M. and Sefat, F. (2018) Effect of TGF- $\beta 1$ on water retention properties of healthy and osteoarthritic chondrocytes. Materials Today: Proceedings, 5(7), pp.15717-15725. 
Raja, T., Mozafari, M., Milan, P., Samadikuchaksaraei, A. and Sefat, F. (2018) Nanoengineered biomaterials for tracheal replacement. Nanoengineered Biomaterials for Regenerative Medicine, pp.285-303.

Sadowska, J. M., Wei, F., Guo, J., Guillem-Marti, J., Ginebra, M. P., \& Xiao, Y. (2018). Effect of nanostructural properties of biomimetic hydroxyapatite on osteoimmunomodulation. Biomaterials, 181, 318-332.

Saranya, N., Saravanan, S., Moorthi, A., Ramyakrishna, B., \& Selvamurugan, N. (2011). Enhanced osteoblast adhesion on polymeric nano-scaffolds for bone tissue engineering. J Biomed Nanotechnol, $7(2), 238-244$.

Scaglione, S., Giannoni, P., Bianchini, P., Sandri, M., Marotta, R., Firpo, G., Valbusa, U., Tampieri, A., Diaspro, A., Bianco, P., \& Quarto, R. (2012). Order versus Disorder: in vivo bone formation within osteoconductive scaffolds. Sci Rep, 2, 274.

Schoolaert, E., Ryckx, P., Geltmeyer, J., Maji, S., Van Steenberge, P. H. M., \& D'Hooge D, R. (2017). Waterborne Electrospinning of Poly(N-isopropylacrylamide) by Control of Environmental Parameters. 9(28), 24100-24110.

Sefat, F. (2017). Fabrication and Characterizations of Hydrogels for Cartilage Repair. Advances in Tissue Engineering \& Regenerative Medicine: Open Access, 2(6).

Sefat, F., Denyer, M. C., \& Youseffi, M. (2011). Imaging via widefield surface plasmon resonance microscope for studying bone cell interactions with micropatterned ECM proteins. J Microsc, 241(3), 282-290.

Sefat, F., Denyer, M. C., \& Youseffi, M. (2014). Effects of different transforming growth factor beta (TGF-beta) isomers on wound closure of bone cell monolayers. Cytokine, 69(1), 75-86.

Sefat, F., Raja, T., Zafar, M., Khurshid, Z., Najeeb, S., Zohaib, S., Ahmadi, E., Rahmati, M. and Mozafari, M. (2018a) Nanoengineered biomaterials for cartilage repair. Nanoengineered Biomaterials for Regenerative Medicine, pp.39-71.

Sefat, F., Raja, T., Moghadam, Z., Milan, P., Samadikuchaksaraei, A. and Mozafari, M. (2018b) Nanoengineered biomaterials for bladder regeneration. Nanoengineered Biomaterials for Regenerative Medicine, pp.459-474.

Sefat, F., Youseffi, M., Khaghani, S., Soon, C. and Javid, F. (2016) Effect of transforming growth factor- $\beta 3$ on mono and multilayer chondrocytes. Cytokine, 83, pp.118-126. 
Sefat, F., Khaghani, S., Nejatian, T., Genedy, M., Abdeldayem, A., Moghaddam, Z., Denyer, M. and Youseffi, M. (2015) Transforming growth factor beta (TGF- $\beta$ ) isomers influence cell detachment of MG-63 bone cells. Tissue and Cell, 47(6), pp.567-574.

Sehaqui, H., Morimune, S., Nishino, T., \& Berglund, L. A. (2012). Stretchable and strong cellulose nanopaper structures based on polymer-coated nanofiber networks: an alternative to nonwoven porous membranes from electrospinning. Biomacromolecules, 13(11), 3661-3667.

Simson, J. A., Strehin, I. A., Lu, Q., Uy, M. O., \& Elisseeff, J. H. (2013). An adhesive bone marrow scaffold and bone morphogenetic-2 protein carrier for cartilage tissue engineering. Biomacromolecules, 14(3), 637-643. and Mechanical Properties of Cellulose Nanofiber Substrates in Aqueous Conditions for Stem Cell Culture. 18(7), 2034-2044.

Soliman, S., Sant, S., Nichol, J. W., Khabiry, M., Traversa, E., \& Khademhosseini, A. (2011). Controlling the porosity of fibrous scaffolds by modulating the fiber diameter and packing density. $J$ Biomed

$840 \quad$ Mater Res A, 96(3), 566-574. acetate fiber mats containing curcumin and release characteristic of the herbal substance. Mozafari, M. (2018) Nanoengineered biomaterials for kidney regeneration. Nanoengineered Biomaterials for Regenerative Medicine, pp.325-344. morphological studies of aligned nanofibrous scaffolds of polycaprolactone fabricated by electrospinning. J Biomater Sci Polym Ed, 17(9), 969-984.

Urbanska, A., Sefat, F., Yousaf, S., Kargozar, S., Milan, P. and Mozafari, M. (2018) Nanoengineered biomaterials for intestine regeneration. Nanoengineered Biomaterials for Regenerative Medicine, pp.363-378. and pore sizes. Biomaterials, 23(8), 1731-1738. 
Wake, M. C., Patrick, C. W., Jr., \& Mikos, A. G. (1994). Pore morphology effects on the fibrovascular tissue growth in porous polymer substrates. Cell Transplant, 3(4), 339-343.

Wang, W., Zhang, P., Zhang, S., Li, F., Yu, J., \& Lin, J. (2013). Structure and properties of novel regenerated cellulose fibers prepared in $\mathrm{NaOH}$ complex solution. Carbohydr Polym, 98(1), 1031-1038.

Wanjare, M., Hou, L., Nakayama, K. H., Kim, J. J., Mezak, N. P., \& Abilez, O. J. (2017). Anisotropic microfibrous scaffolds enhance the organization and function of cardiomyocytes derived from induced pluripotent stem cells. 5(8), 1567-1578.

Wu, L., Magaz, A., Wang, T., Liu, C., Darbyshire, A., Loizidou, M., Emberton, M., Birchall, M., \& Song, W. (2018). Stiffness memory of indirectly 3D-printed elastomer nanohybrid regulates chondrogenesis and osteogenesis of human mesenchymal stem cells. Biomaterials, 186, 64-79.

Yang, G., Li, X., He, Y., Ma, J., Ni, G., \& Zhou, S. (2018). From nano to micro to macro: Electrospun hierarchically structured polymeric fibers for biomedical applications. Progress in Polymer Science, 81, 80-113.

Zarrintaj, P., Bakhshandeh, B., Saeb, M., Sefat, F., Rezaeian, I., Ganjali, M., Ramakrishna, S. and Mozafari, M. (2018) Oligoaniline-based conductive biomaterials for tissue engineering. Acta Biomaterialia, 72, pp.16-34.

Zhang, C., Xue, X., Luo, Q., Li, Y., Yang, K., Zhuang, X., Jiang, Y., Zhang, J., Liu, J., Zou, G., \& Liang, X. J. (2014). Self-assembled Peptide nanofibers designed as biological enzymes for catalyzing ester hydrolysis. ACS Nano, 8(11), 11715-11723.

Zhao, J., Lu, C., He, X., Zhang, X., Zhang, W., \& Zhang, X. (2015). Polyethylenimine-grafted cellulose nanofibril aerogels as versatile vehicles for drug delivery. ACS Appl Mater Interfaces, 7(4), 2607-2615.

Zong, H., Xia, X., Liang, Y., Dai, S., Alsaedi, A., Hayat, T., Kong, F., \& Pan, J. H. (2018). Designing function-oriented artificial nanomaterials and membranes via electrospinning and electrospraying techniques. Mater Sci Eng C Mater Biol Appl, 92, 1075-1091. 